\title{
ABERTURA COMERCIAL E SEUS EFEITOS NA TRANSMISSÃO DE PREÇOS ENTRE OS MERCADOS DE TRIGO ARGENTINO E INTERNACIONAL
}

\author{
Eliane Pinheiro de Sousa ${ }^{1}$ \\ Airton Lopes Amorim² \\ Daniel Arruda Coronel ${ }^{3}$
}

Resumo: Este trabalho buscou examinar os efeitos da abertura comercial na transmissão de preços entre os mercados de trigo argentino e internacional. Para isso, foram comparados dois subperíodos, sendo o primeiro anterior à política de liberação comercial de 1990 e o segundo, pós-liberalização. Essa divisão possibilitou verificar como esse processo alterou as condições de comércio dessa commodity entre os países, sendo a análise realizada por meio dos testes de raiz unitária, cointegração de Johansen, estimação da função impulso-resposta, decomposição da variância dos erros de previsão, teste de exogeneidade e análise do modelo de correção de erros. Os resultados indicaram que os choques não-antecipados no preço do trigo argentino foram corrigidos mais rapidamente após a implementação da política macroeconômica de abertura comercial e que os mercados internacionais passaram a ter mais influência tanto na formação de preços do trigo da Argentina quanto em seus próprios mercados.

Palavras-chave: Transmissão de preços; Mercado de Trigo; Séries de tempo. 
SOUZA, E. P; AMORIM, A. L; CORONEL, D. A. Abertura comercial e seus efeitos na transmissão de ...

\title{
COMMERCIAL OPENING AND ITS EFFECTS ON PRICE TRANSMISSION BETWEEN THE ARGENTINE WHEAT MARKET AND THE INTERNATIONAL WHEAT MARKET
}

\begin{abstract}
This paper aimed to examine the effects of commercial opening on price transmission between the Argentine and international wheat markets. Thus, two sub-periods were compared: the first, the period before the 1990 commercial liberation policy and the second, post-liberation. This division permitted to assess how this process altered the trade conditions of this commodity among countries. Analysis was carried out by means of unitary root tests, Johansen co-integration, impulse-response function estimate, error prediction variance decomposition, exogeneity tests and error correction model analysis. The results indicated that the non-anticipated shocks in Argentine wheat price were corrected more rapidly after implementation of the macroeconomic policy of commercial opening and that the international markets became more influent both on the formation of Argentine wheat prices and their own markets.
\end{abstract}

Keywords: Price transmission; Wheat market; Time series

JEL: C32; Q13

\section{LIBERALIZACIÓN DEL COMERCIO Y SUS EFECTOS SOBRE LA TRANSMISIÓN DE PRECIOS ENTRE LOS MERCADOS DE TRIGO ARGENTINO E INTERNACIONAL}

\begin{abstract}
Resumen: Este trabajo buscó examinar los efectos de la liberalización del comercio en la transmisión de precios entre los mercados de trigo argentino e internacional. Para ello, se compararon dos subperíodos, siendo el primero anterior a la política de liberalización comercial de 1990 y el segundo, después de la liberalización. Esta división posibilitó verificar como ese proceso alteró las condiciones del comercio de esa commodity entre los países, siendo el análisis realizado por medio de testes de raíz unitaria, cointegración de Johansen, apreciación de la función impulso-respuesta, descomposición de la variación de los errores de previsión, test de exogeneidad y análisis del modelo de corrección de errores. Los resultados indicaron que los choques no antecipados en el precio del trigo argentino fueron corregidos más rapidamente
\end{abstract}


después de la implantación de la política macroeconómica de liberalización comercial y que los mercados internacionales pasaron a tener más influencia tanto en la formación de precios del trigo como en sus propios mercados.

Palabras-clave: Transmisión de precios; Mercado de Trigo; Series de tiempo

\section{Introdução}

O trigo é disseminado por todo o mundo, cuja importância na economia mundial não se restringe apenas ao volume de produção, mas à agregação de valor que ocorre ao longo das cadeias de produção e transformação (TOMASINI; AMBROSI, 1998). Na economia argentina, a cadeia tritícola vem cada vez mais aumentando a sua relevância, visto que atualmente esse país representa o quinto maior exportador mundial de trigo. Além disso, este setor tem sido fundamental para a geração de divisas e empregos, conforme a Secretaría de Agricultura, Ganadería, Pesca y Alimentación (SAGPyA, 2009).

Assim como outros produtos da agropecuária argentina, o trigo passou por profundas mudanças, nas últimas décadas, que alteraram a dinâmica produtiva e de comércio deste produto. De acordo com Bisang (2001), os principais fatores da mudança ocorrida na agropecuária argentina foram a incorporação de novas culturas; a adoção de novos processos tecnológicos; a liberação dos preços e a eliminação de várias barreiras de retenção à comercialização. Ainda de acordo com Bisang (2001), ao reduzir as barreiras ao comércio internacional e permitir maior proximidade econômica entre os países, a abertura econômica introduz modificações nos custos de produção dos produtos agropecuários, modifica a característica final destes produtos e altera a formação dos preços dos mesmos.

Diante desse contexto de mudanças e, em especial, das mudanças na formação de preços dos produtos agropecuários, este trabalho avalia os efeitos da abertura comercial, ocorrida na Argentina, nos anos de 1990, sobre o processo de transmissão de preços entre os mercados de trigo argentino e internacional. Estão subjacentes a essa preocupação as perguntas: a) em que nível de mercado se originam as variações nos preços e em que sentido essas variações se transmitem aos demais mercados; b) durante que período se dá a transmissão e com qual intensidade; e c) como a abertura comercial afetou o processo de transmissão de preços.

Para responder a essas questões, analisaram-se as séries de preços do trigo nestes dois mercados, ao longo dos anos de 1980 a 2010, por entender que o comportamento das mesmas pode responder a mudanças na política econômica e a outras mudanças importantes.

As análises de transmissão de preços pretendem responder às seguintes 
questões: a) em que nível de mercado se originam as variações nos preços e em que sentido essas variações se transmitem; b) durante que período se dá a transmissão e com qual intensidade; e c) se existe ou não assimetria na transmissão de preços.

Ademais, é importante destacar que não se encontrou na literatura econômica estudos que busquem captar os efeitos da abertura comercial sobre o processo de transmissão de preços entre os mercados de trigo argentino e internacional. Os estudos desenvolvidos sobre essa temática concentram-se apenas em avaliar a interdependência de preços do trigo entre diferentes países, como, por exemplo, Lema e Brescia (1998), Thompson e Bohl (1999), Donoso, Larson e Thompson (2001), Listorti (2008) e Machado e Freitas (2009).

Vale observar ainda que, a contribuição deste trabalho deve-se não ao fato de ser ou não o primeiro a analisar os efeitos da política de abertura comercial sobre a transmissão de preços do trigo, mas sim por analisar os efeitos de uma política macroeconômica (na época apregoada como um importante passo no processo de desenvolvimento dos países latino americanos), sobre um dos produtos mais importantes das exportações argentinas.

Este trabalho está estruturado em três seções, além desta introdução. Os procedimentos metodológicos e a fonte de dados encontram-se na segunda seção, a apresentação e discussão dos resultados obtidos estão mostrados na terceira seção e, por fim, são destacadas as principais conclusões do estudo.

\section{Metodologia}

\subsection{Transmissão de preços entre mercados espacialmen- te separados}

A transmissão de preços de um produto, entre mercados espacialmente separados, diz respeito à relação de interdependência entre os preços desse produto, quando observado em diferentes mercados. Um exemplo dessa interdependência pode ser verificado por meio do preço internacional e nacional de uma determinada commodity (medido em uma moeda de referência), ou entre o preço ao produtor e ao consumidor de um bem de consumo.

Este princípio tem suas raízes na chamada Lei do Preço Único, que estabelece que sob livre concorrência e na ausência de custos de transporte e barreiras ao comércio, bens homogêneos devem ser comercializados pelo mesmo preço entre dois mercados espacialmente separados, quando seus preços são cotados 
em termos da mesma moeda (KRUGMAN; Obstfeld, 2005)4.

O mecanismo que garante, no longo prazo, a equalização dos preços é a arbitragem. Por meio desse mecanismo, os mercados com menores preços serão induzidos ao aumentar os mesmos, em decorrência da elevação na demanda, e os que apresentam maiores preços tenderão a reduzi-los, devido ao aumento da oferta. Tal processo ocorre até que esses preços se tornem iguais nos dois países (ARDENI, 1989).

Do que foi exposto, pode-se verificar, então, que o conceito de transmissão de preços está alicerçado em três pressupostos, a saber: a) movimento de preços e ajustamento integral, o qual implica transmissão completa em algum ponto de tempo; b) dinâmica e velocidade de ajustamento; e c) assimetria de respostas, ou seja, se as variações de preços são transmitidas assimetricamente entre mercados. (BALCOMBE e MORRINSON, 2002)

As idéias descritas nos parágrafos anteriores são expressas, mais formalmente, pelo modelo teórico de Mundlak e Larson (1992). De acordo com Barbosa, Margarido e Nogueira Junior (2002), no modelo de Mundlak e Larson (1992), o preço doméstico de dado produto $\left(\boldsymbol{P}_{\text {it }}\right)$ é representado como função do seu próprio preço no mercado externo ( $\left.P_{i t}^{*}\right)$, da taxa de câmbio nominal ( $E_{t}$ ) e de tarifas ou subsídios $\left(S_{i t}\right)$ :$$
P_{i t}=P_{t}^{*} E_{t} S_{t}
$$

Essa formulação desconsidera diferenças na qualidade e na estocagem do produto, no custo de transporte, no custo de marketing e em todos os outros insumos domésticos não comercializáveis.

Multiplicando-se os dois lados da equação (1) por, $\frac{1}{E_{t}}$ obtém-se:

$$
P_{i t}^{*} \$=P_{t}^{*} S_{t}
$$

em que: $\boldsymbol{P}_{\boldsymbol{i} \boldsymbol{\alpha}}^{\boldsymbol{\alpha}} \mathbf{\$}$ referem-se aos preços domésticos cotados em dólares.

Para incorporar efeitos de variáveis não incluídas nessa equação, um termo de erro, denotado por $u$, é acrescentado à equação (1), sendo a mesma reescrita, em termos logarítmicos, da seguinte forma:

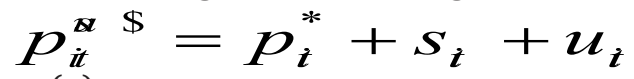

(2)

4 Apesar de sua vasta utilização, a LPU recebe várias críticas. Fackler e Goodwin (2001) e Miljkovic (1999) mostram que os pressupostos teóricos da LPU são muito restritivos, pois não incluem outras variáveis tais como custos de transação e volume negociado entre mercados, os quais são relevantes nas análises de integração e transmissão de preços. 
em que $u \sim I I D\left(\mathrm{~ms}^{2}\right)$ e $E(\boldsymbol{s} \boldsymbol{u})=E\left(p^{*} u\right)=0$, ou seja, $u_{i t}$ não é correlacionado com nenhuma das variáveis explicativas do modelo.

Em sua forma mais simples, o modelo econométrico para a formação do preço doméstico pode ser representado por:

$p_{i t}^{\Delta}=a+b p_{i}^{*}+e_{i}$,

(3)

em que: $p_{i t}^{\alpha}$ é o logaritmo do preço doméstico do produto $i$ no ano $t$ (cotado em Dólar); no presente trabalho, é a representação do preço do trigo argentino; $p_{j t}$ é o logaritmo do preço internacional do produto $i$ no ano $t$ ; no presente trabalho, é a representação do preço internacional do trigo; a constante (ou intercepto); e $\mathbf{b}$ é a elasticidade de transmissão de preço entre esses países.

Sob o parâmetro b são levantadas algumas hipóteses, a serem testadas após a estimação do mesmo. Se o valor de $\mathbf{b}$ for igual à unidade, variações de preço no mercado internacional de trigo serão plenamente transmitidas para o mercado de trigo argentino. Em contrapartida, se o valor de b for igual a zero, tem-se que variações de preço no mercado internacional não influenciarão o processo de formação de preço do trigo argentino.

\subsection{Relação de longo prazo entre as séries de preço do trigo argentino e internacional}

Como a transmissão de preços pressupõe uma relação de longo prazo entre as séries de preços analisadas, o possível relacionamento entre as mesmas foi testado por meio da tradicional metodologia de cointegração.

Vale observar que, como o objetivo deste trabalho era verificar os efeitos da política de abertura comercial na Argentina, sobre a transmissão de preços do trigo, entre o mercado argentino e internacional, a análise de cointegração, bem como os testes de raiz unitária foram realizados para o período como um todo (1980 à 2010) e para os dois subperíodos 1980 a 1990 e 1990 a 2010.

No primeiro caso, buscou-se verificar a existência da transmissão de preços do trigo entre o mercado argentino e o internacional. Ao dividir a amostra em subperíodos e realizar uma estimativa individual para cada um deles, procurou-se comparar a velocidade e o nível da transmissão de preços, antes e depois da política macroeconômica de redução às barreiras comerciais. 


\subsubsection{Testes de raiz unitária}

Para que se possa aplicar a metodologia de cointegração é necessário que as séries sejam não estacionárias, mais especificamente, espera-se que as mesmas sejam integradas de ordem um. Dessa forma, a ordem de integração das séries de preços foi verificada por meio dos testes de raiz unitária de Dickey-Fuller Aumentado (ADF), Phillips-Perron (PP) e KPSS, desenvolvido por Kwiatkowski-Phillips-Schmidt-Shin ${ }^{5}$.

O teste de raiz unitária de Dickey-Fuller Aumentado (ADF) permite verificar a existência ou não de raízes unitárias nas séries temporais, ou seja, se as variáveis são ou não estacionárias (DICKEY e FULLER, 1979, 1981) ${ }^{6}$. Este teste consiste na estimação da seguinte equação por Mínimos Quadrados Ordinários e pode ser expresso, conforme Enders (1995), da seguinte forma:

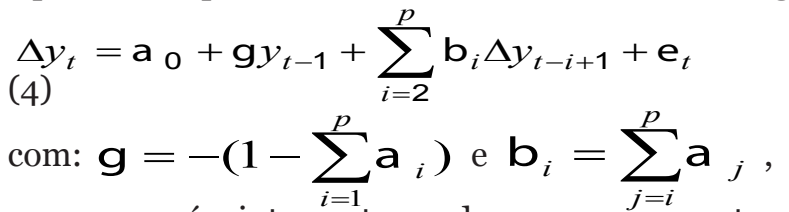

em que: $\mathrm{a}_{0}$ é o intercepto; $\mathrm{g}$ descreve o comportamento da série temporal; $y$ representa a variável dependente; $\Delta$ é a representação do operador de diferença e $\mathrm{e}_{t}$ denota o erro, que se assume ser identicamente e independentemente distribuída. Para determinar o número de defasagens utilizadas no teste para eliminar a autocorrelação residual, utilizou-se o menor valor do critério de Schwarz (SBC).

O parâmetro de interesse nas regressões (sem intercepto e sem tendência; com intercepto; com intercepto e tendência) é $\mathbf{g}$, sendo que, se $\mathbf{g}=\mathbf{0}$ , a série contém uma raiz unitária. Nesse teste, compara-se o resultado da estatística $\mathrm{t}$ com os valores apropriados reportados por Dickey-Fuller para determinar se aceita ou se rejeita a hipótese nula $\mathbf{g}=\mathbf{0}$. A hipótese nula será rejeitada se o valor calculado da estatística $t$ for maior do que o valor crítico de Dickey-Fuller, indicando que a série é estacionária; caso contrário, a série é não-estacionária.

O teste de Phillips-Perron (PP) consiste em uma alternativa não-paramétrica ao teste $\mathrm{ADF}$. Esse teste pode ser empregado quando os resíduos forem serialmente correlacionados. Nesse caso, a hipótese adotada no teste de Dickey-Fuller de que o erro seja identicamente distribuído é desconsiderada (PHILLIPS; PERRON, 1988).

A série de preços é considerada estacionária em nível, ou seja, não possui

5 A utilização de diferentes testes de raiz unitária permite verificar a robustez quanto à conclusão da estacionaridade ou não das variáveis em consideração. Isso é feito por meio da comparação dos resultados obtidos pelos diferentes testes.

6 Um processo estocástico é estacionário quando a sua média e a sua variância são constantes ao longo do tempo e quando o valor da covariância entre dois períodos de tempo depende apenas da distância, do intervalo ou da defasagem entre os períodos de tempo, e não do próprio tempo em que a covariância é calculada. 
uma raiz unitária se a hipótese nula puder ser rejeitada; caso contrário, a série é não-estacionária.

Outro teste indicado na literatura econômica como complementar do teste de Dickey-Fuller, porém mais robusto é o teste KPSS, desenvolvido por Kwiatkowski-Phillips-Schmidt-Shin. Esse teste diferencia-se dos demais, visto que estabelece como hipótese nula a estacionariedade da série (KWIATKOWSKI et al., 1992).

No teste KPSS, a variável dependente $y$ pode ser constituída apenas pela presença de um termo constante ou formada pelo intercepto acrescido por um componente de tendência determinística. Essa série é dita estacionária se a hipótese nula for aceita, ou seja, quando a estatística do teste for menor que seus respectivos valores críticos.

\subsubsection{Análise de cointegração ${ }^{7}$}

Para verificar a cointegração entre as variáveis foi utilizado o procedimento de Johansen (1988). Este procedimento considera todas as variáveis como endógenas e sua utilização não é limitada pela existência de endogeneidade do regressor (relação causal no sentido da variável dependente para a variável explicativa), sendo que o mesmo utiliza Máxima Verossimilhança para estimar os vetores de cointegração e permite testar e estimar a presença de vários vetores e não só de um único vetor de cointegração.

De acordo com Harris (1995), definido um vetor $z_{t}$ de $n$ variáveis potencialmente endógenas, é possível especificar o seguinte processo gerador, e modelar $z_{t}$ como um Vetor Auto-regressivo (VAR) irrestrito com k defasagens de $z_{t}$ :

$$
z_{t}=A_{1} z_{t-1}+\ldots+A_{k} z_{t-k}+u_{t}
$$

em que: $z_{t}$ é um vetor (nx1), $A_{i}$ é uma matriz de parâmetros (nxn) e $u_{t}$ $I I D\left(\mathrm{~ms}^{2}\right)$.

No caso deste artigo, o termo z refere-se aos preços do trigo no mercado argentino e internacional.

Ainda conforme Harris (1995) e substituindo o vetor z pelos preços do trigo, a equação (5) pode ser reparametrizada em termos de um modelo Vetorial de Correção de Erro (VEC) esboçado como:

7 Como observado por Greene (2008), mesmo que variáveis individuais não sejam estacionárias, mas exista pelo menos uma combinação linear estacionária das variáveis, então pode-se afirmar que essas variáveis são cointegradas. Isso significa que existe uma relação de equilíbrio de longo prazo entre elas, que pode ser estimada e analisada. 
$\Delta P_{t}=\Gamma_{1} \Delta P_{t-1}+\ldots+\Gamma_{k-1} \Delta P_{t-k+1}+\prod P_{t-k}+\mathrm{e}_{t}$

(6)

e m que: $\quad \Gamma_{i}=-\left(I-A_{1}-\cdots-A_{i}\right), \quad(\mathrm{i}=1, \ldots, \mathrm{k}-1)$ e $\Pi=-\left(I-A_{1}-\cdots A_{k}\right)$. Da forma como especificado, o sistema contém informações de curto e longo prazo a mudanças de $P_{t}$, via estimativas de $\hat{\boldsymbol{\Gamma}}_{i}$ e $\hat{\boldsymbol{\Pi}}$, respectivamente, onde $\boldsymbol{D}=\boldsymbol{a}^{\boldsymbol{a}^{\prime}}$, com $\alpha$ representando a velocidade de ajustamento ao desequilíbrio e $\beta$ a matriz de coeficientes de longo prazo.

Supondo que k seja igual a unidade, o termo $\prod P_{t-1}$ pode ser expresso por: $\prod_{t-1}=\left[\begin{array}{l}\mathrm{a}_{1} \\ \mathrm{a}_{2}\end{array}\right]\left[\mathrm{b}_{1} \mathrm{~b}_{2}\left[\begin{array}{l}\text { Pit } \\ P_{t}^{*}\end{array}\right]_{t-1}=\left[\begin{array}{l}\mathrm{a}_{1} \\ \mathrm{a}_{2}\end{array}\right]\left[\mathrm{b}_{1} \text { Pit } \mathrm{b}_{2} P_{t}^{*}\right]_{-1}\right.$ (7) Pit e $P_{i t}^{*}$ representa, respectivamente, os preços do trigo argentino e internacional, conforme indicado na equação (3).

Seguindo o exemplo demonstrado por Sampaio et al. (2005), normalizando para o coeficiente de Pit e multiplicando b por $1 / b_{1}$ e a por $b_{1}$, a equação (6) pode ser reescrita da seguinte forma:

$$
\Delta \text { Pit }_{t}=\Gamma_{1} \Delta \text { Pit }_{t-1}+\Gamma_{\mathrm{p}} \Delta \text { Pit }^{*}{ }_{t-1}+\mathrm{a}_{1} \mathrm{~b}_{1}\left[\text { Pit }_{t-1}+\left(\mathrm{b}_{2} / \mathrm{b}_{1}\right) P i t^{*}{ }_{t-1}\right]+\mathrm{e}_{t}
$$

A importância do modelo de correção de erro reside no fato de permitir a ligação entre aspectos relacionados à dinâmica de curto prazo com os de longo prazo (HAMILTON, 1994). Dessa forma, os mecanismos de correção de erro pretendem fornecer um caminho para combinar as vantagens de se modelar tanto em nível quanto nas diferenças. Nesse modelo, tanto a dinâmica do processo de ajustamento de curto prazo quanto de longo prazo são modeladas simultaneamente.

O número de vetores de cointegração depende do posto ou rank ( $r$ ) da matriz $\Pi$. Em termos de vetores de cointegração, têm-se três possibilidades, conforme Enders (1995): se o posto de $\Pi$ é completo, então as variáveis $Y_{t}$ são I(o), ou seja, significa que qualquer combinação linear entre as variáveis é estacionária e o ajuste do modelo deve ser efetuado com as variáveis em nível; se o posto de $\Pi$ é zero, então não há relacionamento de cointegração e o modelo deve ser ajustado com as variáveis em diferença e, quando $\Pi$ tem posto reduzido, há $r$ vetores de cointegração.

Para Enders (1995), o rank de uma matriz é igual ao número de raízes características estritamente diferentes de zero, que pode ser identificado por meio de dois testes estatísticos. O primeiro deles é o teste do traço, que testa a hipótese nula de que o número de vetores de cointegração distintos é menor ou igual a r contra a hipótese alternativa de que o número desses vetores é maior do que $\mathrm{r}$. 
Que pode ser definido por:

I $_{(9)}^{\text {trace }}(r)=-T \sum_{i=r+1}^{n} \ln \left(1-\mathbf{I}_{i}\right)$

em que I ${ }_{i}=$ valores estimados das raízes características obtidos da matriz $\Pi ; T=$ número de observações.

O segundo teste é o do máximo autovalor, que testa a hipótese nula de que o número de vetores de cointegração é $r$ contra a hipótese alternativa de existência de $r+1$ vetores de cointegração, podendo ser expresso por:

$$
I_{\max }(r, r+1)=-T \ln \left(1-I_{r+1}^{\prime}\right)
$$

Verificada a cointegração entre as séries analisadas, estima-se o modelo VEC, conforme definido nas expressões (6 e 8).

\subsubsection{Teste de hipóteses sobre os parâmetros}

Para verificar quais mercados fazem parte do equilíbrio de longo prazo e se a integração entre eles pode ser considerada perfeita, confirmando-se assim a hipótese da Lei do Preço Único (LPU), foram realizados testes de hipóteses sobre os parâmetros $\alpha$ e $\beta$, respectivamente ${ }^{8}$.

Seguindo a notação de Harris (1995), os testes de hipóteses sobre os parâmetros $b$ seguem a seguinte forma:

$$
H_{1}: \mathrm{b}=H \mathrm{j},
$$

em que $H$ representa uma matriz de dimensões ( $p x s)$ e $s$ representa o número de coeficientes b que não estão restritos. A matriz j é uma matriz ( $s x r$ ) de parâmetros a serem estimados envolvendo $r$ vetores de cointegração.

Neste trabalho, testam-se as seguintes hipóteses nulas $\left(H_{0}\right)$

$\beta_{\text {ARGE }}=0$

(12)

$\beta_{\text {INTER }}=0$

(13)

8 Barbosa, Margarido e Nogueira Junior (2002) e Coelho (2004) também utilizam desses expedientes em seus trabalhos, vindo dos mesmos a motivação para realização destes testes neste trabalho. 
$\beta_{\text {ARGE }}=\beta_{\text {INTER }}$

(14)

As hipóteses nulas descritas em (12) e (13) objetivam verificar se o preço do mercado de trigo argentino e internacional podem ser considerados integrados no período analisado. Já a hipótese (14) visa testar o grau de integração entre os mercados de preço de trigo argentino e internacional.

A estatística do teste razão de verossimilhança aplicado sobre os parâmetros $\beta$ pode ser expressa da seguinte forma:

$$
-2 \log Q\left(H_{0}\right)=-T \sum_{i=1}^{r} \log \left[\frac{1-\left.\right|_{i} ^{*}}{\left(1-\left.\right|_{i} ^{\wedge}\right.}\right] \sim \dot{\div}_{r(n-m)}^{2}
$$

em que $T$, número de observações; $r$, número de vetores de cointegração; $\hat{\imath}_{i}{ }^{*}$ e $\hat{\imath}_{i}$ autovalores do modelo restrito e irrestrito e, $n$, número de variáveis.

Posteriormente, empregaram-se os testes de hipótese sobre os parâmetros $\alpha$. A significância desse teste indica que a variável preço do trigo no mercado considerado não é exogenamente fraca em relação ao parâmetro de longo prazo. A presença de exogeneidade fraca significa que a variável não reage ao desequilíbrio de equilíbrio de longo prazo.

Neste trabalho, testam-se as seguintes hipóteses nulas $\left(H_{0}\right)$ :

$\alpha_{\mathrm{ARGE}}=0$

(16)

$\alpha_{\text {INTER }}=0$

(17)

$\alpha_{\text {ARGE }}=\alpha_{\text {INTER }}$

As hipóteses nulas (16) e (17) testam a exogeneidade nos mercados de trigo argentino e internacionais, e a hipótese (18) testa se a velocidade de resposta das variáveis a um dado desequilíbrio de curto prazo no processo de ajuste 
de longo prazo é a mesma no mercado argentino e no internacional. A estatística de teste de razão de verossimilhança do parâmetro $\alpha$ segue a mesma formulação definida para o parâmetro $b$.

\subsection{Fontes de dados}

Os dados de preços do trigo argentino (PARGE) foram coletados no site da Secretaria de Agricultura, Ganadería, Pesca y Alimentación ( $\underline{\text { SAGPyA) e os }}$ preços internacionais (PINTER) foram coletados através do site do Instituto de Pesquisa Econômica Aplicada (IPEA), para o período de janeiro de 1980 a março de 2010. Esse período de análise foi dividido em dois subperíodos, com o primeiro anterior à política de liberação comercial de 1990 e o segundo, pós-liberalização.

Com relação aos dados empregados, também é relevante destacar que esses preços não foram deflacionados, pois, conforme Wang e Tomek (2007) e Siqueira (2007), o deflacionamento incorpora uma tendência nos dados, logo não faz sentido incluir uma tendência antes da realização do teste de cointegração.

\section{Resultados e discussões}

\subsection{Análise para o período completo (1980 a 2010)}

O comportamento dos preços do trigo na Argentina e no mercado internacional pode ser visualizado na Figura 1. Nota-se que os preços do trigo na Argentina e do mercado internacional não apresentaram uma tendência linear bem definida ao longo do tempo. 

CADO INTERNACIONAL (LPINTER), JANEIRO DE 1980 A MARÇO DE 2010.

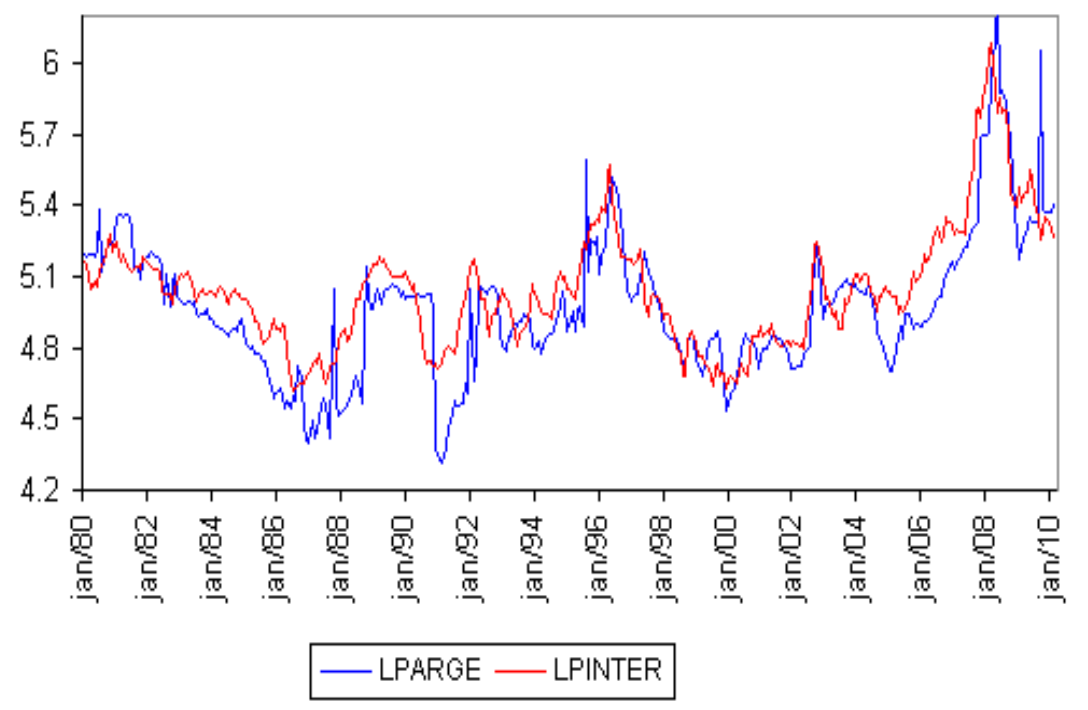

A Figura 1 mostra, ainda, que as séries de preços mensais do trigo movimentam-se conjuntamente no período analisado. Logo, aparentemente, essas séries seriam cointegradas, ou seja, haveria uma relação de equilíbrio de longo prazo entre elas. Entretanto, essa hipótese deve ser verificada de forma mais criteriosa, o que foi realizado por meio dos testes econométricos de cointegração.

Para verificar se as séries analisadas são estacionárias, realizou-se o teste de Dickey-Fuller Aumentado (ADF) e de Phillips Perron (PP). Conforme os resultados mostrados na Tabela 1, ambos os testes indicam que as séries de preços do trigo da Argentina e do mercado internacional não são estacionárias em nível, visto que os valores calculados da estatística $t$ são menores, em módulo, que seus respectivos valores críticos a 1\%, em todos os modelos analisados. Em outros termos, a hipótese nula de raiz unitária não pode ser rejeitada para estas séries. Entretanto, observa-se que essas séries passam a ser estacionárias em primeira diferença, sinalizando que apenas uma diferenciação é suficiente para torná-las estacionárias. 
SOUZA, E. P; AMORIM, A. L; CORONEL, D. A. Abertura comercial e seus efeitos na transmissão de ...

TABELA 1 - RESULTADOS DO TESTE DE ADF E PHILLIPS PERRON EM NÍVEL E EM PRIMEIRA DIFERENÇA PARA AS SÉRIES MENSAIS DE PREÇOS LOGARITMIZADAS DO TRIGO DA ARGENTINA (LPARGE) E DO MERCADO INTERNACIONAL (LPINTER), JANEIRO DE 1980 E MARÇO DE 2010

\begin{tabular}{|c|c|c|c|}
\hline Séries & Modelos $^{\text {(a) }}$ & Estatísticas do & Estatísticas do teste \\
\hline LPARGE & & $\begin{array}{c}\text { teste ADF } \\
-0,0317 \\
-2,8069 \\
-3,1402\end{array}$ & $\begin{array}{c}\text { Phillips Perron } \\
-0,0200 \\
-3,3572 \\
-3,7055\end{array}$ \\
\hline LPINTER & & $\begin{array}{l}-0,14509 \\
-2,5658 \\
-2,8729\end{array}$ & $\begin{array}{l}-0,0537 \\
-2,4627 \\
-2,7743 \\
\end{array}$ \\
\hline $\begin{array}{l}\text { DLPARGE } \\
\text { (b) }\end{array}$ & & $\begin{array}{l}-25,1371 \\
-25,1031 \\
-25,0932\end{array}$ & $\begin{array}{l}-25,2552 \\
-25,2209 \\
-25,2166\end{array}$ \\
\hline $\begin{array}{l}\text { DLPINTER } \\
\text { (b) }\end{array}$ & III & $\begin{array}{l}-14,6156 \\
-14,5956 \\
-14,5837\end{array}$ & $\begin{array}{l}-14,6046 \\
-14,5845 \\
-14,5730\end{array}$ \\
\hline
\end{tabular}

FONTE: Dados da pesquisa.

(a) $\mathrm{O}$ modelo I corresponde ao modelo sem intercepto e sem tendência e seu valor crítico ao nível de significância de 1\% é -2,5714; o modelo II, ao modelo com intercepto e sem tendência e seu valor crítico ao nível de significância de $1 \%$ é -3,4482; e o modelo III, ao modelo com intercepto e com tendência e seu valor crítico ao nível de significância de 1\% é -3,9837.

(b) DLPARGE e DLPINTER indicam, respectivamente, primeira diferença das séries Preços do Trigo Argentino e Preço Internacional de Trigo.

Para aumentar a robustez dos resultados dos testes de raiz unitária obtidos, empregou-se o teste KPSS, que tem como hipótese nula a estacionariedade das séries. Os resultados deste teste são apresentados na Tabela 2, os quais indicam que todas as séries são estacionárias em primeira diferença para os dois tipos de modelos analisados. Portanto, constata-se que as séries de preços estudadas são integradas de ordem um.

TABELA 2 - RESULTADOS DO TESTE DE KPSS EM PRIMEIRA DIFERENÇA PARA AS SÉRIES MENSAIS DE PREÇOS LOGARITMIZADAS DO TRIGO DA ARGENTINA (LPARGE) E DO MERCADO INTERNACIONAL, JANEIRO DE 1980 A MARÇO DE 2010

\begin{tabular}{ccc}
\hline Séries & \multicolumn{2}{c}{ Modelos $^{(a)}$} \\
\cline { 2 - 3 } DLPARGE & I & II \\
\hline DLPINTER & 0,0725 & 0,0567 \\
\hline
\end{tabular}

FONTE: Resultados da pesquisa

(a) $\mathrm{O}$ modelo I corresponde ao modelo com intercepto e sem tendência e seu valor crítico ao nível de significância de 1\% é o,7390; e o modelo II, ao modelo com intercepto e com tendência e seu valor crítico ao nível de significância de $1 \%$ é 0,2160 . 
$\mathrm{O}$ fato de elas serem integradas de mesma ordem constitui um pré-requisito para examinar se as séries são cointegradas, o que é identificado por meio do teste de Johansen com o intuito de verificar se elas possuem relacionamento de longo prazo. Entretanto, antes disso, deve-se determinar o número de defasagens mais adequado para a estimação do modelo VAR. Seguindo os critérios de Akaike (AIC), Schwarz (SC) e Hannan-Quinn (HQ), o modelo VAR deve possuir duas defasagens.

Em se tratando do modelo VAR, é importante apresentar e discutir os resultados relativos às funções de impulso-resposta e à decomposição da variância dos erros de previsão. A Tabela 3 mostra os resultados das elasticidades da função de impulso-resposta, indicando as respostas das variáveis LPARGE e LPINTER a um dado impulso sobre a própria variável e sobre a outra. Os dados indicam que um desvio padrão em LPARGE, no período atual, causará, respectivamente, o,0064 e 0,0559 desvios padrões sobre ela própria e sobre LPINTER, no décimo segundo mês. Esta mesma interpretação pode ser feita para os choques de LPINTER sobre LPARGE e sobre si mesma.

TABELA 3 - ELASTICIDADES DA FUNÇÃO DE IMPULSO-RESPOSTA DE LPARGE E LPINTER, JANEIRO DE 1980 A MARÇO DE 2010

\begin{tabular}{ccc|c|c}
\hline \multirow{2}{*}{\begin{tabular}{c} 
Período \\
\cline { 2 - 5 }
\end{tabular}} & \multicolumn{2}{c}{$\begin{array}{c}\text { Efeitos de choques de LPARGE } \\
\text { sobre }\end{array}$} & \multicolumn{2}{c}{$\begin{array}{c}\text { Efeitos de choques de } \\
\text { LPINTER sobre }\end{array}$} \\
\cline { 2 - 5 } & LPARGE & LPINTER & \multicolumn{1}{c}{ LPARGE } & LPINTER \\
\hline 1 & 0,1042 & 0,0000 & $-0,0017$ & 0,0513 \\
3 & 0,0470 & 0,0305 & 0,0017 & 0,0654 \\
6 & 0,0211 & 0,0526 & 0,0027 & 0,0591 \\
9 & 0,0108 & 0,0576 & 0,0028 & 0,0524 \\
12 & 0,0064 & 0,0559 & 0,0027 & 0,0467 \\
\hline
\end{tabular}

FONTE: Resultados da pesquisa.

As elasticidades da função impulso-resposta de LPARGE e LPINTER indicam que um choque não antecipado sobre os preços do trigo no mercado da Argentina ou no mercado internacional gera pequenos efeitos no curto prazo. Dessa forma, verifica-se que tanto no mercado interno (Argentina) quanto no externo (Internacional), os desequilíbrios transitórios são corrigidos lentamente.

Com relação à decomposição da variância dos erros de previsão de LPARGE, a Tabela 4 mostra que, após doze meses da incidência de um choque não antecipado sobre essa variável, 41,34\% da decomposição da variância dos erros de previsão devem-se a ela própria, sendo o restante atribuído à variável LPINTER, o que indica uma forte influência de LPINTER sobre LPARGE. Quanto à decomposição da variância dos erros de previsão de LPINTER, os resultados indicam que, após doze meses do choque inicial não antecipado 
sobre essa variável, 99,81\% do seu comportamento é decorrente dela própria, sendo que 0,19\% deve-se à LPARGE.

TABELA 4 - DECOMPOSIÇÃO DA VARIÂNCIA DOS ERROS DE PREVISÃO EM PERCENTAGEM DE LPARGE E LPINTER, JANEIRO DE 1980 A MARÇO DE 2010

\begin{tabular}{ccc|c|c}
\hline Período & \multicolumn{2}{c}{$\begin{array}{c}\text { Decomposição da variân- } \\
\text { cia dos erros de previsão } \\
\text { de LPARGE }\end{array}$} & \multicolumn{2}{c}{$\begin{array}{c}\text { Decomposição da variância dos } \\
\text { erros de previsão de LPINTER }\end{array}$} \\
\cline { 2 - 5 } & LPARGE & LPINTER & LPARGE & LPINTER \\
\hline 1 & 100, oooo & 0,0000 & 0,1076 & 99,8924 \\
3 & 92,8684 & 7,1316 & 0,0650 & 99,9350 \\
6 & 69,9047 & 30,0952 & 0,1147 & 99,8853 \\
9 & 51,9624 & 48,0376 & 0,1571 & 99,8429 \\
12 & 41,3360 & 58,6640 & 0,1869 & 99,8131 \\
\hline
\end{tabular}

FONTE: Resultados da pesquisa.

Assim, verifica-se que os erros de previsão são mais explicados pelos choques internacionais. Resultados semelhantes foram encontrados por Machado e Freitas (2009), que estudaram transmissão de preços do trigo entre países do Mercosul e Estados Unidos.

A partir da visualização gráfica, mostrada na Figura 1, verifica-se que aparentemente as duas séries se movimentam conjuntamente no tempo e a diferença entre as mesmas é estável. Para verificar econometricamente se essas séries de preços apresentam relacionamento de equilíbrio de longo prazo, empregouse o teste de Johansen. Os resultados dos testes do traço e do máximo autovalor são apresentados na Tabela 5 .

TABELA 5 - RESULTADOS DO TESTE DE COINTEGRAÇÃO DE JOHANSEN PARA AS SÉRIES MENSAIS DE PREÇOS DO TRIGO DA ARGENTINA E DO MERCADO INTERNACIONAL DE JANEIRO DE 1980 A MARÇO DE 2010

\begin{tabular}{ccccc}
\hline $\begin{array}{c}\text { Hipótese } \\
\text { nula }\end{array}$ & $\begin{array}{c}\text { Teste do } \\
\text { Traço }\end{array}$ & $\begin{array}{c}\text { Valor Crítico } \\
(5 \%)\end{array}$ & $\begin{array}{c}\text { Teste do máxi- } \\
\text { mo autovalor }\end{array}$ & $\begin{array}{c}\text { Valor Crítico } \\
(5 \%)\end{array}$ \\
\hline $\mathrm{r}=\mathrm{O}$ & $58,0954^{*}$ & 12,3209 & $58,0937^{*}$ & 11,2248 \\
$\mathrm{r} \leq 1$ & 0,0017 & 4,1299 & 0,0017 & 4,1299 \\
\hline
\end{tabular}


FONTE: Resultados da pesquisa.

* Indica rejeição da hipótese nula a 5\% de significância.

Os testes do traço e do máximo autovalor permitiram rejeitar a hipótese nula de que não há nenhum vetor de cointegração ao nível de $5 \%$ de significância. Dessa forma, as séries de preço do trigo nesse período avaliado contêm um único vetor de cointegração. Os resultados do estudo desenvolvido por Donoso et al. (2001) também indicaram que os preços do trigo entre Argentina e Estados Unidos foram cointegrados para o período entre 1982 a 1999.

A equação de cointegração é dada por LPARGE $=0,9852$ LPINTER, o que significa que, ceteris paribus, $98,52 \%$ das variações de preços do trigo no longo prazo, ocorridas no mercado internacional, foram transmitidas para o preço do trigo na Argentina. Com base nesse resultado da elasticidade de transmissão do preço, observa-se a predominância da Lei do Preço Único nesses mercados, já que seu coeficiente foi bem próximo da unidade. Entretanto, conforme Baulch (1997), a simples existência de um vetor de cointegração não pode ser considerada condição suficiente para determinar a perfeita integração de mercado, nem para garantir a participação de todas as séries no equilíbrio de longo prazo. Portanto, é necessário realizar testes de hipóteses sobre os parâmetros $\beta$ para se testar sua significância e a interação entre os preços.

Os resultados desse teste encontram-se na Tabela 6. A partir desses dados, pode-se rejeitar a hipótese nula sobre os parâmetros $\mathrm{b}_{A R G E} \mathrm{e} \mathrm{b}_{\text {INTER }}$. Isto significa que os movimentos de preços no mercado argentino e no mercado internacional são significativamente relevantes no estabelecimento do padrão de equilíbrio de longo prazo. Assim, esses mercados podem ser considerados integrados, já que choques ocorridos em um mercado são repassados para o outro mercado no longo prazo.

Tendo em vista que esses mercados são integrados, testou-se a hipótese de perfeita integração entre os mesmos. Esta hipótese foi rejeitada, indicando que a alteração de preços em um mercado não é completamente transmitida ao outro mercado no longo prazo, constatando-se, dessa forma, que a Lei do Preço Único não é perfeitamente verificada para o mercado de trigo no período analisado. 
SOUZA, E. P; AMORIM, A. L; CORONEL, D. A. Abertura comercial e seus efeitos na transmissão de ...

TABELA 6 - TESTES DE SIGNIFICÂNCIA DE RESTRIÇÃO SOBRE O PARÂMETRO DE LONGO PRAZO (B) DO VETOR DE COINTEGRAÇÃO

\begin{tabular}{ccc}
\hline Hipótese nula & Razão de Verossimilhança & Valor crítico (5\%) \\
\hline $\mathrm{b}_{A R G E}=0$ & $58,0566^{*}$ & 3,84 \\
$\mathrm{~b}_{I N T E R}=0$ & $58,0914^{*}$ & 3,84 \\
$\mathrm{~b}_{\text {ARGE }}=\mathrm{b}_{\text {INTER }}$ & $58,0851^{*}$ & 3,84 \\
\hline
\end{tabular}

FONTE: Resultados da pesquisa.

* Indica rejeição a 5\% de significância.

Os valores dos testes de hipótese sobre o parâmetro $\alpha$ estão mostrados na Tabela 7. Tais estimativas indicam que a hipótese de exogeneidade fraca é rejeitada na série de preços do trigo na Argentina. Isso significa que os níveis de preços do trigo vigentes na Argentina reagem a desequilíbrios transitórios ocorridos nos níveis de preços desse cereal no mercado internacional. $\mathrm{O}$ mesmo não acontece com os preços do trigo no mercado internacional, cuja hipótese se mostra exogenamente fraca em relação ao equilíbrio de longo prazo. Esses resultados confirmam as expectativas de que os preços do trigo no mercado internacional influenciam seus níveis de preços na Argentina.

Quanto à hipótese nula $\mathrm{a}_{A R G E}=\mathrm{a}_{I N T E R}$, constata-se que as velocidades de resposta das variáveis a uma dada situação de desequilíbrio de curto prazo no processo de ajuste de longo prazo não são estatisticamente iguais para o mercado argentino e o mercado internacional de trigo no período considerado.

TABELA 7 - TESTES DE SIGNIFICÂNCIA DE RESTRIÇÃO SOBRE O PARÂMETRO A

\begin{tabular}{ccc}
\hline Hipótese nula & Razão de Verossimilhança & Valor crítico (5\%) \\
\hline $\mathrm{a}_{A R G E}=0$ & $58,0078^{*}$ & 3,84 \\
$\mathrm{a}_{I N T E R}=0$ & 0,4385 & 3,84 \\
$\mathrm{a}_{\text {ARGE }}=\mathrm{a}_{\text {INTER }}=0$ & $49,3931^{*}$ & 3,84 \\
\hline
\end{tabular}

FONTE: Resultados da pesquisa.

* Indica rejeição a 5\% de significância.

Com base nos resultados da Tabela 8, pode-se inferir que 30,01\% do desequilíbrio de curto prazo referente à trajetória de longo prazo são corrigidos a cada mês, indicando que precisaria em média três meses e dez dias para corrigir 
o desequilíbrio, ou seja, que esses desequilíbrios transitórios são corrigidos num prazo relativamente rápido. Ademais, uma variação de $1 \%$ no preço do trigo na Argentina no mês anterior causará uma variação no mês atual de $16,30 \%$ em seus preços. O mesmo raciocínio pode ser interpretado quanto à variação do preço do trigo no mercado internacional, ou seja, uma variação de $1 \%$ no preço do trigo no mercado internacional propiciará um acréscimo de 27,06\% em seus preços no mês seguinte.

TABELA 8 - ESTIMAÇÃO DO VEC REFERENTE À VARIÁVEL PREÇO DO TRIGO NA ARGENTINA E PREÇO DO TRIGO NO MERCADO INTERNACIONAL, JANEIRO DE 1980 A MARÇO DE 2010

\begin{tabular}{ccc}
\hline Variáveis & LPARGE $_{\mathrm{t}}$ & $\Delta$ LPINTER \\
\hline $\mathrm{a}$ & $-0,3001$ & 0,0124 \\
& {$[-7,8998](0,03800)$} & {$[0,6597](0,0188)$} \\
$\Delta$ LPARGE $_{\mathrm{t}-1}$ & $-0,1630$ & 0,0147 \\
& {$[-3,3850](0,0482)$} & {$[0,6169](0,0239)$} \\
$\Delta$ LPINTER $_{\mathrm{t}-1}$ & 0,0280 & 0,2706 \\
& {$[0,2494](0,1124)$} & {$[4,8580](0,0557)$} \\
\hline
\end{tabular}

Fonte: Resultados da pesquisa.

Nota: Os valores entre colchetes referem-se à estatística t e os valores entre parênteses correspondem ao erro padrão.

\subsection{Análise para os subperíodos (1980 a 1990 e 1990 a} 2010)

Os resultados discutidos na seção anterior contemplam a série de preços mensais do trigo nos mercados argentinos e internacionais no período entre janeiro de 1980 a março de 2010. Entretanto, no início dos anos 1990, a maioria dos países da América Latina passou pelo processo de abertura comercial. Nesse sentido, é importante investigar se essa política macroeconômica interferiu o processo de transmissão de preços do trigo entre Argentina e mercados internacionais. Para tal, é importante replicar os testes econométricos considerando os subperíodos antes da abertura comercial (janeiro de 1980 a fevereiro de 1990) e pós-abertura comercial (a partir de março de 1990 a março de 2010).

Para verificar se as séries de preços do trigo são estacionárias nesses subperíodos avaliados, repetiram-se os cálculos dos testes de raiz unitária ADF, PP e KPSS para cada um desses subperíodos e confirmaram-se que essas séries de preços do trigo são estacionárias em primeira diferença. Feito isto, emprega- 
se o teste de cointegração de Johansen, porém deve-se primeiro identificar o número de defasagens que deve ser incluído no modelo VAR para cada subperíodo. Com base no critério de Schwarz, considerou-se uma defasagem no modelo VAR no primeiro subperíodo e duas defasagens no segundo subperíodo. Vale destacar que no teste de cointegração foi considerada uma defasagem a menos do que a definida no modelo VAR.

Os resultados do teste de cointegração de Johansen (Tabela 9) indicam que se rejeita a hipótese nula de que não há vetor de cointegração nos dois subperíodos analisados. Em ambas as situações, os testes sinalizam a presença de um vetor de cointegração.

TABELA 9 - RESULTADOS DO TESTE DE COINTEGRAÇÃO DE JOHANSEN PARA AS SÉRIES MENSAIS DE PREÇOS DO TRIGO DA ARGENTINA (LPARGE) E DO MERCADO INTERNACIONAL (LPINTER) NOS DOIS SUBPERÍODOS ANALISADOS

\begin{tabular}{cccccc}
\hline Períodos $^{(\mathrm{a})}$ & $\begin{array}{c}\text { Hipótese } \\
\text { nula }\end{array}$ & $\begin{array}{c}\text { Teste do } \\
\text { Traço }\end{array}$ & $\begin{array}{c}\text { Valor } \\
\text { Crítico } \\
(5 \%)\end{array}$ & $\begin{array}{c}\text { Teste do máxi- } \\
\text { mo autovalor }\end{array}$ & $\begin{array}{c}\text { Valor } \\
\text { Crítico } \\
(5 \%)\end{array}$ \\
\hline Subperíodo 1 & $\mathrm{r}=0$ & $21,0749^{(\mathrm{b})}$ & 12,3209 & $21,0115^{(\mathrm{b})}$ & 11,2248 \\
\hline Subperíodo 2 & $\mathrm{r} \leq 1$ & 0,0634 & 4,1299 & 0,0634 & 4,1299 \\
\hline & $\mathrm{r}=0$ & $45,0046^{(\mathrm{b})}$ & 12,3209 & $45,0042^{(\mathrm{b})}$ & 11,2248 \\
& $\mathrm{r} \leq 1$ & 0,0004 & 4,1299 & 0,0004 & 4,1299 \\
\hline
\end{tabular}

FONTE: Resultados da pesquisa.

(a) O subperíodo 1 compreende o período de janeiro de 1980 a fevereiro de 1990; e o subperíodo 2 contempla o período de março de 1990 a março de 2010.

(b) Indica rejeição da hipótese nula a 5\% de significância.

A equação de cointegração do período que antecede a abertura comercial é expressa por: LPARGE = 0,9812 LPINTER e a equação de cointegração relativa ao período pós-abertura comercial é dada por: LPARGE = 0,9870 LPINTER. A elasticidade de transmissão do preço do trigo entre os mercados estudados apresenta um ligeiro acréscimo após a abertura comercial.

Como o coeficiente de elasticidade de transmissão do preço do trigo foi próximo à unidade nos dois subperíodos analisados, verifica-se que a Lei do Preço Único prevalece nos mercados de trigo nesses períodos. No entanto, para confirmar essa evidência, torna-se necessário proceder aos testes de hipóteses sobre os parâmetros $\beta$. Refazendo esses testes para os dois subperíodos analisados, obtiveram-se resultados idênticos ao do período completo, isto 
é, os preços do trigo nos mercados argentinos e internacionais participam das relações de longo prazo e rejeita-se a hipótese nula de perfeita integração em tais mercados, logo a Lei do Preço Único não é perfeitamente verificada nesses mercados de trigo analisados.

Os resultados do teste de hipótese $\alpha$ para o período completo mostraram que os níveis de preços do trigo vigentes na Argentina reagem a desequilíbrios transitórios ocorridos nos níveis de preços desse cereal no mercado internacional, porém o inverso não se verifica. Esse resultado não se altera quando se analisa cada subperíodo separado, ou seja, a abertura comercial não influencia a hipótese de exogeneidade fraca, que é rejeitada para a série de preços do trigo na Argentina. Ademais, da mesma forma que obtida no período completo de análise, as velocidades de resposta das variáveis a uma dada situação de desequilíbrio de curto prazo no processo de ajuste de longo prazo não são estatisticamente iguais para os mercados de trigo em ambos os subperíodos avaliados.

Como as séries de preços do trigo são cointegradas, conforme verificado mediante a Tabela 9, tais séries convergem para uma condição de equilíbrio de longo prazo, tornando-se relevante a estimação do Modelo de Correção de Erro (VEC). A Tabela 10 apresenta os resultados da estimação do VEC para cada subperíodo analisado. Tais resultados indicam que a remoção dos desequilíbrios de curto prazo requereu menos tempo após a abertura comercial, ou seja, os desequilíbrios de curto prazo referente à trajetória de longo prazo necessitavam, em média de três meses e meio para serem corrigidos antes da abertura comercial e pós-abertura, três meses passaram a ser suficientes para corrigir esses desequilíbrios.

TABELA 10 - ESTIMAÇÃO DO VEC REFERENTE À VARIÁVEL PREÇO DO TRIGO NA ARGENTINA (LPARGE) E PREÇO DO TRIGO NO MERCADO INTERNACIONAL (LPINTER) NOS DOIS SUBPERÍODOS CONSIDERADOS

\begin{tabular}{cccc}
\hline Períodos $^{(a)}$ & Variáveis & $\Delta$ LPARGE $_{+}$ & $\Delta$ LPINTER $_{+}$ \\
\hline Subperíodo 1 & a & $-0,2856$ & $-0,0062$ \\
& & {$[-4,7697](0,0599)$} & {$[-0,2764](0,0224)$} \\
Subperíodo 2 & a & $-0,3326$ & 0,0129 \\
& & {$[-6,9927](0,0476)$} & {$[0,5071](0,0254)$} \\
\hline
\end{tabular}

FONTE: Resultados da pesquisa.

Nota: ${ }^{(a)}$ O subperíodo 1 compreende o período de janeiro de 1980 a fevereiro de 1990; e o subperíodo 2 contempla o período de março de 1990 a março de 2010.

Os valores entre colchetes referem-se à estatística t e os valores entre parên- 
teses correspondem ao erro padrão.

Ademais, também se estimou novamente as funções de impulso-resposta e a decomposição da variância dos erros de previsão para cada subperíodo analisado. Esses resultados encontram-se nas Tabelas 11 e 12. Os resultados das elasticidades da função de impulso-resposta (Tabela 11) mostram que um desvio padrão em LPARGE, no período atual, causará o,0187 e 0,004 desvios padrões sobre ela mesma no décimo segundo mês, respectivamente, antes e após a abertura comercial, ao passo que gerará o,0372 e o,0619 desvios padrões sobre LPINTER, antes e após a abertura comercial. Essa análise pode ser replicada para os choques de LPINTER sobre LPARGE e sobre ela própria. Tais números evidenciam que os desequilíbrios transitórios são corrigidos lentamente mesmo após a abertura comercial.

TABELA 11 - ELASTICIDADES DA FUNÇÃO DE IMPULSO-RESPOSTA DE LPARGE E LPINTER NOS DOIS SUBPERÍODOS CONSIDERADOS

\begin{tabular}{|c|c|c|c|c|}
\hline \multirow[t]{2}{*}{ Período } & \multicolumn{4}{|c|}{ Efeitos de choques de LPARGE sobre } \\
\hline & LPARGE $^{\text {(a) }}$ & LPINTER $^{\text {(a) }}$ & LPARGE $^{(\mathrm{b})}$ & $\begin{array}{c}\text { LPINTER } \\
\text { (b) }\end{array}$ \\
\hline 1 & 0,0949 & 0,0000 & 0,1087 & 0,0000 \\
\hline 3 & 0,0439 & 0,0226 & 0,0454 & 0,0352 \\
\hline 6 & 0,0254 & 0,0384 & 0,0161 & 0,0602 \\
\hline 9 & 0,0208 & 0,0395 & 0,0048 & 0,0649 \\
\hline 12 & 0,0187 & 0,0372 & 0,0004 & 0,0619 \\
\hline
\end{tabular}

Efeitos de choques de LPINTER sobre

LPARGE $^{(a)}$ LPINTER $^{(a)}$ LPARGE ${ }^{(b)}$ LPINTER

(b)

\begin{tabular}{ccccc}
\hline 1 & 0,0023 & 0,0362 & $-0,0038$ & 0,0574 \\
\hline 3 & 0,0063 & 0,0400 & $-0,0002$ & 0,0751 \\
6 & 0,01139 & 0,0301 & $-0,0015$ & 0,0698 \\
9 & 0,0119 & 0,0255 & $-0,0021$ & 0,0622 \\
12 & 0,0113 & 0,0231 & $-0,0021$ & 0,0549 \\
\hline
\end{tabular}

FONTE: Resultados da pesquisa. 
SOUZA, E. P; AMORIM, A. L; CORONEL, D. A. Abertura comercial e seus efeitos na transmissão de ...

TABELA 12 - DECOMPOSIÇÃO DA VARIÂNCIA DOS ERROS DE PREVISÃO EM PERCENTAGEM DE LPARGE E LPINTER NOS DOIS SUBPERÍODOS CONSIDERADOS

\begin{tabular}{|c|c|c|c|c|}
\hline \multirow[t]{2}{*}{ Período } & \multicolumn{4}{|c|}{ Decomposição da variância dos erros de previsão de LPAR- } \\
\hline & LPARGE $^{(a)}$ & LPINTER $^{\text {GE }}$ & LPARGE $^{(\mathrm{b})}$ & LPINTER $^{(b)}$ \\
\hline 1 & 100,0000 & 0,0000 & 100,0000 & 0,0000 \\
\hline 3 & 95,2841 & 4.7159 & 01.3448 & 8.6552 \\
\hline 6 & 78,0162 & 21,9838 & 64,4611 & 35,5389 \\
\hline 9 & 65,0426 & 34,9574 & 45,6684 & 54,3316 \\
\hline 12 & 57,2602 & 42.7398 & 35.5560 & \\
\hline \multirow[t]{2}{*}{ Período } & \multicolumn{4}{|c|}{ Decomposição da variancia dos erros de previsão de LPIN- } \\
\hline & LPARGE $^{(a)}$ & 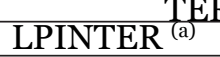 & LPARGE $^{(b)}$ & LPINTER $^{(b)}$ \\
\hline 1 & 0,4001 & 99,5999 & 0,4373 & 99,5627 \\
\hline 3 & 1,0269 & 98,9731 & 0,1040 & 99,8960 \\
\hline 6 & 4,3394 & 95,6606 & 0,0633 & 99,9367 \\
\hline 9 & 7,2190 & 92,7810 & 0,0713 & 99,9287 \\
\hline 12 & 9,1216 & 90,8784 & 0,0834 & 99,9167 \\
\hline
\end{tabular}

FONTE: Resultados da pesquisa.

Nota: (a) O subperíodo 1 compreende o período de janeiro de 1980 a fevereiro de 1990; e o subperíodo 2 contempla o período de março de 1990 a março de 2010.

Pela análise da decomposição da variância da variável LPARGE, verifica-se uma substituição na decomposição dos erros de previsão dessa variável, em que $57,26 \%$ e $42,74 \%$ de seu comportamento, decorrido doze meses após um choque não-antecipado sobre essa variável, antes da abertura comercial, eram atribuídos, respectivamente, a ela própria e à LPINTER; passando para $35,56 \%$ e $64,44 \%$, respectivamente, após a abertura comercial. Em outros termos, com a implementação dessa política macroeconômica de liberalização comercial, há uma maior participação de LPINTER na explicação dos erros de previsão de LPARGE e dela mesma. Os dados também mostram um acréscimo da participação de LPINTER na explicação da variância dos erros de previsão dela própria pós-abertura comercial.

\section{Conclusões}

As inferências realizadas neste trabalho demonstraram a existência de relações de equilíbrio de longo prazo entre os preços do trigo argentino e internacional, ou seja, as variáveis são cointegradas.

A partir da implementação da política macroeconômica de abertura comercial, 
os choques não-antecipados no preço do trigo argentino foram corrigidos mais rapidamente, o que pode ser atribuído à maior integração desse mercado com os mercados internacionais de trigo.

Ademais, verificou-se que, no período pós-liberalização, os mercados internacionais passaram a ter mais influência tanto na formação de preços do trigo da Argentina quanto em seus próprios mercados.

Por fim, é relevante destacar que este trabalho aferiu os efeitos da abertura comercial sobre o grau de integração dos mercados de trigo argentino e internacional, considerando apenas seus preços, porém outros fatores, como cotações da taxa de câmbio, podem ser incorporados em trabalhos futuros.

\section{Referências}

ARDENI, P. G. (1989). "Does the Law of One Price really hold for commodity prices?” American Journal of Agricultural Economics 71 (3): 661-669.

BALCOMBE, B. K.; MORRISON, J. (2002). Commodity price transmission: a critical of techniques and an application to select tropical export commodities. Roma: FAO.

BARBOSA, M. Z.; MARGARIDO, M. A.; NOGUEIRA JUNIOR, S. (2002). “Análise da elasticidade de transmissão de preços no mercado brasileiro de algodão”. Nova Economia, 12 (2):79-108.

BAULCH, B. (1997). “Test for food Market integration revisited". The Journal of Development Studies, 33 (4):512-534.

BISANG, R. (2001). Apertura económica, innovación y estructura productiva: la aplicación de biotecnología en la producción agrícola pampeana argentina, Buenos Aires: Universidad Nacional de General Sarmiento.

BUENO, R. D. L. D. (2008). Econometria de séries temporais. São Paulo: Cengage Learning.

COELHO, A. B. (2004). “A cultura do algodão e a questão da integração entre preços internos e externos”. Revista de Economia e Sociologia Rural 42 (1): 153-169.

DICKEY, D. A.; FULLER, W. A. (1979). “Distribution of the estimators for autoregressive time series with a unit root”. Journal of the American Statistical Association, 74 (366): 427-431.

DICKEY, D. A.; FULLER, W. (1981). “A Likelihood ratio statistics for autoregressive time series with a unit root”. Econometrica, 49: 1057-1073.

DONOSO, C. I.; LARSON, D. W.; THOMPSON, S. R. (2001). Regional Market Integration and MERCOSUR: Implications for wheat trade. In: International Food and Agribusiness Management Association Meeting World Food and Agribusiness Symposium. Sydney, Australia.

ENDERS, W. (1995). Applied Econometric Time Series. Nova York: John Wiley \& Sons. 
SOUZA, E. P; AMORIM, A. L; CORONEL, D. A. Abertura comercial e seus efeitos na transmissão de ...

ENGLE, R. F.; GRANGER, C. W. (1987). “Co-integration and error-correction: representation, estimation and testing”. Econometrica 55 (2): 251-276.

FACKLER, P. L.; GOODWIN, B. K. (2001). "Spatial price analysis”. In: GARDNER, B. L.; RAUSSER, G. C.; (eds.). Handbook of Agricultural Economics. Amsterdam: Elsevier.

GREENE, W. H. (2008). Econometrics Analysis. New Jersey: Pearson Education.

HAMILTON, J. D. (1994). Time series analysis. New Jersey: Princeton University Press.

HARRIS, R.I.D. (1995). Using cointegration analysis in econometric modelling. London: Prentice-Hall-Harvester Wheatsheaf.

INSTITUTO DE PESQUISA ECONÔMICA APLICADA (IPEA). (2009). Base de Dados. 2009. Disponível em: <http://www.ipeadata.gov.br/ipeaweb.dll/ipeadata $>$.

JOHANSEN, S. (1988). "Statistical analysis of cointegration vectors". Journal of Economic Dynamic and Control 12: 231-254.

KRUGMAN, P. R.; OBSTFELD, M. (2005). International economics: theory and policy. Massachusetts: Addson Welsley.

KWIATKOWSKI, D. et al. (1992). “Testing the null hypothesis of stationarity against the alternative of a unit root. How sure are we that economic time series have a unit root?” Journal of Econometrics 54: 159-178.

LEMA, D.; BRESCIA, V. (1998). La convergencia de los precios agricolas de la Argentina y de Los EE. UU. La "ley de un solo precio" para los commodities pampeanos. Documento de Trabajo 2. Disponível em: <http:// www.inta.gov. ar/ies/docs/doctrab/DT 02.pdf>. Acesso em: 29 nov., 2009.

LISTORTI, G. (2008). International price transmission on soft wheat markets: which role for policy variables in cointegration relationships? In: XII Congress of the European Association of Agricultural Economists, Ghent, Belgium.

MACHADO, T. D. A.; FREITAS, C. A. (2009). Transmissão de preços do trigo entre países do Mercosul e Estados Unidos. In: ILHA, A. D.; FREITAS, C. A. (Orgs.). O agronegócio brasileiro e o comércio internacional. Curitiba: Editora CRV.

MILJKOVIC, D. (1999). “The law of one price in international trade: a critical review”. Review of Agricultural Economics 21 (1): 126-139.

MUNDLAK; Y; LARSON, D. F. (1992). "On the transmission of world agricultural prices”. The World Bank Economic Review 6 (3): 399-422.

PHILLIPS, P.C.B.; PERRON, P. (1988). "Testing for a unit root in time series regression”. Biometrika 75 (2): 335-346.

SAMPAIO, L. M. B et al. (2005). "Inter-relação das exportações de Brasil, Argentina e EUA: uma abordagem utilizando autoregressão vetorial”. In: XLIII Congresso Brasileiro de Economia e Sociologia Rural, Anais. Ribeirão Preto, CD-ROM.

SECRETARÍA DE AGRICULTURA, GANADERÍA, PESCA Y ALIMENTACIÓN (SAGPyA). (2009). Agricultura. 2009. Disponível em: < http://www.sagpya. mecon.gov.ar>. Acesso em: 29 nov., 2009.

SIQUEIRA, K. B. (2007). The dynamics of farm milk price formation in Brazil. 
Viçosa: Tese de Doutorado, Universidade Federal de Viçosa, Programa de PósGraduação em Economia Aplicada.

THOMPSON, S. R.; BOHL, M. T. (1999). International wheat price transmission and CAP Reform. In: Conference AAEA Annual Meeting. Disponível em:<http:// sard.ruc.edu.cn/INTERNATIONAL\%20WHEAT\%20PRICE\%20TRANSMISSION>. Acesso em: 29 nov., 2009.

TOMASINI, R. G. A.; AMBROSI, I. (1998). “Aspectos econômicos da cultura do trigo”. Cadernos de Ciência e Tecnologia 15 (2): 59-84.

WANG, D; TOMEK, W. G. “Commodity prices and unit root tests”. American Journal of Agricultural Economics 89 (4): 873-889. 